\title{
A GENERAL RAMSEY PRODUCT THEOREM
}

\author{
R. L. GRAHAM AND J. H. SPENCER
}

\begin{abstract}
Call a family $\mathscr{F}$ of subsets of a set $U$ Ramsey if no partition of $U$ into finitely many parts can split every $F \in \mathscr{F}$. We show that under very general conditions an arbitrary collection of Ramsey families in fact has a much stronger uniform Ramsey property.
\end{abstract}

A family $\mathscr{F}$ of finite subsets of a set $U$ is said to be Ramsey if for all integers $r<\infty$ and all mappings $\chi: U \rightarrow\{1,2, \ldots, r\} \equiv[1, r]$, there exists an $F \in \mathscr{F}$ which is homogeneous, i.e., such that for some $i \in[1, r] F \subseteq$ $\chi^{-1}(i)$. Given an arbitrary mapping $P: U \times U \rightarrow U$, a family $\mathcal{F}$ is said to be a $P$-ideal of $U$ if

$$
F \in \mathscr{F} \Rightarrow P(F, u) \in \mathcal{F}, \quad P(u, F) \in \mathscr{F},
$$

for all $u \in U$, where $P$ is extended to $2^{U} \times 2^{U}$ in the usual way, i.e., for $X$, $Y \subseteq U$

$$
P(X, Y) \equiv\{P(x, y): x \in X, y \in Y\} .
$$

The following somewhat unexpected result shows that the Ramsey property holds simultaneously for arbitrary collections of Ramsey families under quite general conditions.

THEOREM. Let $\left\{\mathscr{F}_{\alpha}\right\}_{\alpha \in A}$ be an arbitrary family of Ramsey $P$-ideals of $U$ where $P: U \times U \rightarrow U$ is arbitrary. Then for any $r<\infty$ and any mapping $\chi$ : $U \rightarrow[1, r]$, there exists $i \in[1, r]$ and $F_{\alpha} \in \mathscr{F}_{\alpha}, \alpha \in A$, such that $F_{\alpha} \subseteq \chi^{-1}(i)$ for all $\alpha \in A$.

Proof. We first show by induction that for any integer $m$ and any finite subcollection $\mathscr{F}_{\alpha_{1}}, \ldots, \mathscr{F}_{\alpha_{1}}$ of $\left\{\mathscr{F}_{\alpha}\right\}_{\alpha \in A}$, there is a finite set $F=$ $\left[\mathscr{F}_{\alpha_{1}}, \ldots, \mathscr{F}_{\alpha_{t}}\right] \subseteq U$ such that for any mapping $\chi: F \rightarrow[1, m]$, there is an $i \in[1, m]$ and $F_{j} \in \mathscr{F}_{\alpha_{j}}$ such that $F_{j} \subseteq \chi^{-1}(i)$ for $1 \leqslant j \leqslant t$. For $t=1$, this follows at once from a well-known compactness principle (see [1]). Let $\bar{t}>1$ be fixed and suppose the assertion holds for all $t<\bar{t}$. Also, the assertion is immediate for $m=1$. Thus, let $\bar{m}>1$ be fixed and suppose the assertion also holds for $t=\bar{t}$ and all $m<\bar{m}$. Let $\mathscr{F}_{\alpha_{1}}, \ldots, \mathscr{F}_{\alpha_{\bar{\tau}}}$ be an arbitrary fixed subcollection of $\left\{\mathscr{F}_{\alpha}\right\}_{\alpha \in A}$. By induction, the sets

$$
X=\left[\mathscr{F}_{\alpha_{1}}, \ldots, \mathscr{F}_{\alpha_{\bar{i}-1}}\right]_{\bar{m}}, \quad Y=\left[\mathscr{F}_{\alpha_{\bar{i}}}\right]_{m^{*}} \text { where } m^{*}=\bar{m}^{|X|}
$$

Received by the editors November 1, 1977 and, in revised form, April 21, 1978.

AMS (MOS) subject classifications (1970). Primary 05A05, $05 \mathrm{~A} 17$.

Key words and phrases. Ramsey's Theorem, partitions. 
and

$$
F^{*}=P(X, Y)
$$

exist and are finite.

Let $\chi: U \rightarrow[1, \bar{m}]$ be an arbitrary fixed mapping of $U$ into $[1, \bar{m}]$. Define a new mapping $\chi^{*}$ on $Y$ so that

iff

$$
\chi^{*}(y)=\chi^{*}\left(y^{\prime}\right), \quad y, y^{\prime} \in Y
$$

$$
\chi(P(x, y))=\chi\left(P\left(x, y^{\prime}\right)\right) \text { for all } x \in X
$$

Since

$$
|P(X, y)| \leqslant|X| \text { for all } y \in Y
$$

then we can take $\chi^{*}$ to be a mapping of $Y$ into $\left[1, m^{*}\right]$. By the definition of $Y$, there exists $F_{\bar{i}} \in \mathscr{F}_{\alpha_{\bar{i}}}$ such that for some $i \in\left[1, m^{*}\right], F_{\bar{i}} \subseteq \chi^{*-1}(i)$. Let $f \in F_{\bar{i}}$.

We now define another mapping $\chi^{\prime}: X \rightarrow[1, \bar{m}]$ by letting

$$
\chi^{\prime}(x)=\chi(P(x, f)), \quad x \in X \text {. }
$$

Note that the value of $\chi^{\prime}$ is actually independent of the choice of $f$.

By the definition of $X$, there exists $k \in[1, \bar{m}]$ and $F_{j} \in \mathscr{F}_{\alpha_{j}}$ such that $F_{j} \subseteq \chi^{\prime-1}(k), 1 \leqslant j \leqslant \bar{t}-1$. Therefore,

$$
P\left(F_{j}, f\right) \subseteq \chi^{-1}(k), \quad 1 \leqslant j \leqslant \bar{t}-1,
$$

and so

$$
P\left(F_{j}, F_{\bar{t}}\right) \subseteq \chi^{-1}(k), \quad 1 \leqslant j \leqslant \bar{t}-1
$$

since

$$
\chi(P(x, f))=\chi\left(P\left(x, f^{\prime}\right)\right), \quad x \in X, \quad f, f^{\prime} \in F_{\bar{i}} .
$$

But

$$
P\left(F_{j}, f\right) \in \mathscr{F}_{\alpha_{j}}, \quad 1 \leqslant j \leqslant \bar{t}-1,
$$

since $F_{\alpha_{j}}$ is a $P$-ideal, and $P\left(x, F_{i}\right) \in \mathscr{F}_{\alpha_{i}}$ for the same reason. Since all $t$ of these sets are in $\chi^{-1}(k)$ then we have shown that $P(X, Y)$ can be taken as $\left[\mathscr{F}_{\alpha_{1}}, \ldots, \mathscr{F}_{\alpha_{\bar{t}}}\right]_{\bar{m}}$. This completes the induction step and the first assertion is proved.

Now, suppose the theorem fails. Thus, for some $r$ there is a mapping $\chi$ : $U \rightarrow[1, r]$ and families $\mathscr{F}_{\beta_{i}} \in\left\{\mathscr{F}_{\alpha}\right\}_{\alpha \in A}$ such that

$$
F_{i} \subseteq \chi^{-1}\{i\} \quad \text { for all } F_{i} \in \mathscr{F}_{\beta_{i}}, \quad 1 \leqslant i \leqslant r .
$$

By the preceding assertion, the (finite) set

$$
\left[\mathscr{F}_{\beta_{1}}, \ldots, \mathscr{F}_{\beta_{r}}\right]_{r} \subseteq U
$$

exists. Thus, for some $k \in[1, r]$ and $F_{j}^{\prime} \in \mathscr{F}_{\beta_{j}}$,

$$
F_{j}^{\prime} \subseteq \chi^{-1}(k), \quad 1 \leqslant j \leqslant r .
$$

In particular, $F_{k}^{\prime} \subseteq \chi^{-1}(k)$ and $F_{k}^{\prime} \in \mathscr{F}_{\beta_{k}}$. This contradicts (1) and the theorem is proved. 
Typical applications of this theorem can produce significant strengthenings of many of the standard Ramsey-type results. For example, an old result of Gallai (see [3]), generalizing the theorem of van der Waerden on arithmetic progressions (see [2], [4]), asserts that for any finite subset $C$ of $\mathbf{E}^{n}$, in any partition of $\mathbf{E}^{n}$ into finitely many classes, some class always contains a subset $C^{\prime}$ which is similar to $C$. Using the product theorem of this note, taking $U$ to be $\mathbf{E}^{n}$ and for $\bar{x}, \bar{y} \in \mathbf{E}^{n}$, defining $P(\bar{x}, \bar{y})=\bar{x}+\bar{y}$, we see, in fact, that in any partition of $\mathbf{E}^{n}$ into finitely many classes, one class must contain similar copies of every finite subset of $\mathbf{E}^{n}$.

By taking $U=\mathbf{Z}^{+}$, the set of positive integers, and $P(x, y)=x y$, we obtain the following classical theorem of Rado [3]. Call a system $\mathcal{S}$ of homogeneous, linear equations regular, if for any partition of $\mathbf{Z}^{+}$into finitely many classes, $\mathcal{S}$ has a solution entirely in one class. (Such systems were completely characterized by Rado.) Then, in fact, by the product theorem, for any partition of $\mathbf{Z}^{+}$into finitely many classes, some class contains solutions to every regular system of equations.

\section{REFERENCES}

1. N. G. de Bruijn and P. Erdös, $A$ colour problem for infinite graphs and a problem in the theory of relations, Indag. Math. 13 (1951), 371-373.

2. R. L. Graham and B. L. Rothschild, $A$ short proof of van der Waerden's theorem on arithmetic progressions, Proc. Amer. Math. Soc. 42 (1974), 385-386.

3. R. Rado, Studien zur Kombinatorik, Math. Z. 36 (1933), 424-480.

4. B. L. van der Waerden, Beweis einer Baudetschen Vermutung, Nieuw Arch. Wisk. 15 (1927), 212-216.

Bell Laboratories, Murray Hill, New Jersey 07974

Department of Mathematics, State University of New York, Stony Brook, New York 11794 